\title{
Craniocerebral aspergillosis in immunocompetent hosts: Surgical perspective
}

Muhammad Shahzad Shamim

Aga Khan University, shahzad.shamim@aku.edu

Arshad A. Siddiqui

Aga Khan University

Ather Enam

Aga Khan University, ather.enam@aku.edu

Ahmed Ali Shah

Aga Khan University

Rashid Jooma

Aga Khan University, rashid.jooma@aku.edu

See next page for additional authors

Follow this and additional works at: https://ecommons.aku.edu/pakistan_fhs_mc_surg_neurosurg

Part of the Neurology Commons, Neurosurgery Commons, and the Surgery Commons

\section{Recommended Citation}

Shamim, M., Siddiqui, A. A., Enam, A., Shah, A. A., Jooma, R., Anwar, S. (2007). Craniocerebral aspergillosis in immunocompetent hosts: Surgical perspective. Neurology India, 55(3), 274-281.

Available at: https://ecommons.aku.edu/pakistan_fhs_mc_surg_neurosurg/264 
Authors

Muhammad Shahzad Shamim, Arshad A. Siddiqui, Ather Enam, Ahmed Ali Shah, Rashid Jooma, and Saleha Anwar

This article is available at eCommons@AKU: https://ecommons.aku.edu/pakistan_fhs_mc_surg_neurosurg/264 


\title{
Craniocerebral aspergillosis in immunocompetent hosts: Surgical perspective
}

\author{
Muhammad Shahzad Shamim, Arshad A. Siddiqui, Syed Ather Enam, Ahmed Ali Shah, \\ Rashid Jooma*, Saleha Anwar** \\ Division of Neurosurgery, The Aga Khan University, *Department of Neurosurgery, Jinnah Postgraduate Medical Centre, ${ }^{*}$ Department of \\ Radiology, Liaquat National Postgraduate Medical Centre, Karachi, Pakistan
}

\begin{abstract}
Craniocerebral aspergillosis is a rare but dangerous variety of central nervous system infections. Surgery is being widely recognized as the cornerstone of management. Due to the rarity of the disease, difficulty and delay in diagnosis and poor outcome, there is very little in the literature regarding the various surgical strategies that may be adopted in these patients. Early aggressive surgery followed by chemotherapy offers the best chances. Surgical planning would depend upon the type and location of the disease process as well as the condition of the patient. Perioperative care holds immense importance and knowledge of possible complications is essential. Aspergillosis of the central nervous system is difficult to diagnose and equally difficult to treat. Surgery remains the cornerstone of management followed by systemic antifungal medications. Results are better in immunocompetent patients as compared to those who are immunocompromised.
\end{abstract}

Key words: Aspergillosis, fungal infections, immunocompetent hosts

Surgical treatment of fungal infections of the central nervous system (CNS) has always been a challenge to the neurosurgeon. The CNS may be involved primarily, as primary intracranial infection or secondarily through hematogenous spread of the disease from elsewhere. Fungal infection can also spread directly from adjacent structures, notably sinonasal disease. Fungal infection of the CNS is extremely dangerous, affecting mainly the immunocompromised hosts with a reported mortality of almost $100 \%$ in earlier literature. Fortunately, the disease is relatively uncommon, accounting for less than five per cent of all CNS infections and was even referred to as "almost always a clinical surprise" in a recent review by Nadkarni and Goel.[1] However, recently we have noticed a rise in the reporting of fungal infections of the CNS. ${ }^{[2-8]}$ The reason for this can be the increasing number of immunocompromised patients due to the growing epidemic of acquired immunodeficiency syndrome (AIDS), chronic granulomatous diseases, autoimmune diseases or due to the increasing population of patients having had solid organ transplants and on immunosuppressants. The reason can also be the widespread availability of neuroimaging i.e. computerized tomography (CT) and MRI scanning which has lead to earlier diagnosis and thus earlier referrals to neurosurgical centers.

Of late a newer class of patients have been recognized, those who are apparently immunocompetent, harboring CNS fungal infections. These cases present an interesting diagnostic dilemma for the neurosurgeons and neuroradiologists, as neither the clinical nor the imaging features of CNS fungal infections are truly diagnostic of the disease and with the patient being immunocompetent, the possibility of fungal infection is considered low on the list of differentials. ${ }^{[9]}$

\section{Pathophysiology}

Aspergillus fumigatus is the most commonly identified human pathogen, especially in immunocompromised hosts. Since Aspergillus spores are commensals in the respiratory tract and external auditory canal, it is understandable why maxillary sinusitis and lungs are the most common sites of primary aspergillus infection. Primary aspergillosis of the paranasal sinus has been classically described to be of two kinds; either noninvasive (also called allergic Aspergillus sinusitis) or invasive. Infection may reach the CNS either through direct spread or hematogenous spread. Erosion of bone is not always necessary for direct intracranial extension as the fungus has the propensity to spread along vessels that serve as direct channels for the seeding of aspergilli. 
Single or multiple abscesses with marked vascular invasion with or without thrombosis is the hallmark of cerebral aspergillosis on pathologic examination. Aspergillus has a tendency to invade vessels producing a sort of necrotizing angiitis leading to secondary thrombosis with or without hemorrhage. Fungal hyphae are found in large, intermediate and small blood vessels with invasion through vascular walls into adjacent tissue. The evolving hemorrhagic infarcts later convert into septic infarcts with associated abscesses and cerebritis. The mechanism of damage at the cellular level in cerebral Aspergillus lesions has been recently shown to be due to secretion of various necrotizing factors with toxic and lytic activity toward neurons and glial cells. ${ }^{[10]}$ Although the mechanism of invasiveness in immunologically competent hosts remains unclear, it is possibly caused by qualitative cellular or sub-cellular immunodeficiency that is either unrecognized or poorly characterized. Nevertheless, formation of granulomas and the presence of epithelioid and multinucleate giant cells in these patients indicate at least some integrity of immune response. Purulent lesions may be chronic and have a tendency towards fibrosis and granuloma formation. In purulent lesions, pus is seen in the centre of the abscesses with abundant polymorphs at the periphery. Granulomas consist of lymphocytes, plasma cells and fungal hyphae. ${ }^{[11]}$ In summary, brain abscess, epidural abscess, vasculitis, stroke-like illness, granulomatous mass and meningitis are the various types of CNS aspergillosis. ${ }^{[12]}$

\section{Presentation}

Classically, craniocerebral aspergillosis should be considered in any patient known or suspected to be immunocompromised, presenting with signs, symptoms and radiological features of space-occupying lesion with or without a preceding history of nasal blockade/ discharge. Multiple studies have described a variety of presenting symptoms such as nasal stuffiness, headache, peri-orbital pain, vomiting, convulsions, hemiparesis, cranial nerve deficits (diplopia/anosmia), proptosis, sensory impairment, dysarthria, lethargy, impaired consciousness and seizures with or without fever and rarely, ear discharge. ${ }^{[3,13,14]}$ In patients with paranasal sinus disease orbital extension with proptosis, ophthalmoplegia, visual deterioration (which may lead to total monocular blindness) and chemosis may occur. Another feature usually associated with these patients is hypertelorism. Features typical of meningitis and subarachnoid hemorrhage resulting from mycotic aneurysms may also manifest. ${ }^{[11,15]}$ The disease is usually slowly progressive and symptoms may persist for months. Alternatively, patients may also present acutely with cerebral infarctions or hemorrhage, a manifestation of vascular invasion of the fungus. ${ }^{[16]}$ Aspergillosis has been shown to have a tendency for invasion of the walls of both small and large blood vessels, which may lead to either thrombosis and subsequent infarction or equally dangerous hemorrhage. The organism can also spread beyond the vessel walls and form abscesses in the altered brain tissue.

\section{Radiological Diagnosis}

The findings in the conventional CT scan and magnetic resonance (MR) imaging are frequently minimal or absent in the hyperacute stage of CNS aspergillosis. ${ }^{[17,18]}$ On CT scanning, a number of features have been reported, though none are specific for fungal disease. Presence of a hyperdense mass lesion in the sinuses along with bony expansion or erosion of sinus walls and/or cranial base is frequently observed in patients [Figures 1 and 2] having intracranial aspergillosis of sinonasal origin i.e. the invasive variety of aspergillosis. ${ }^{[2,12]}$ Abscesses demonstrate ring or homogenous enhancement along with mass effect. At times there may be slight or

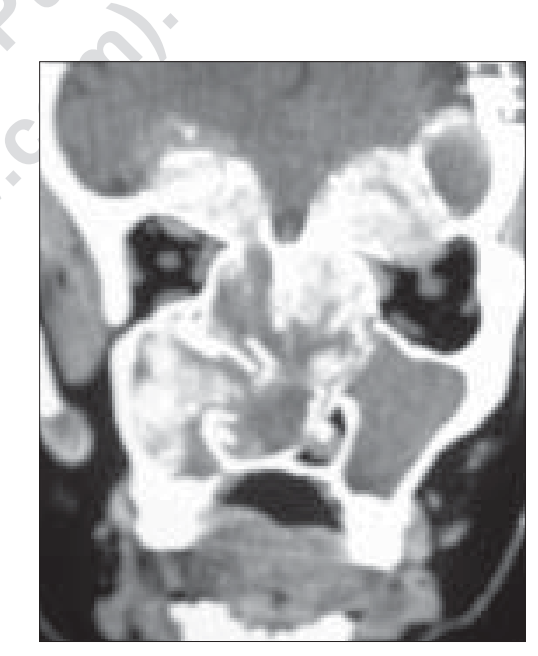

Figure 1: CT scan coronal sections showing Aspergillus involvement of paranasal sinuses with intra-cranial extension, typical of invasive aspergillosis. Patient was immunocompetent

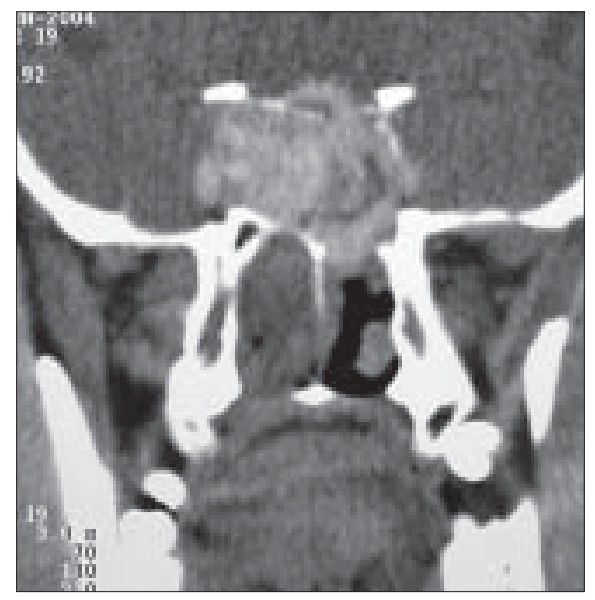

Figure 2: CT scan coronal section showing sinonasal disease eroding the cribiform plate and extending intracrially. Patient was immunocompetent 

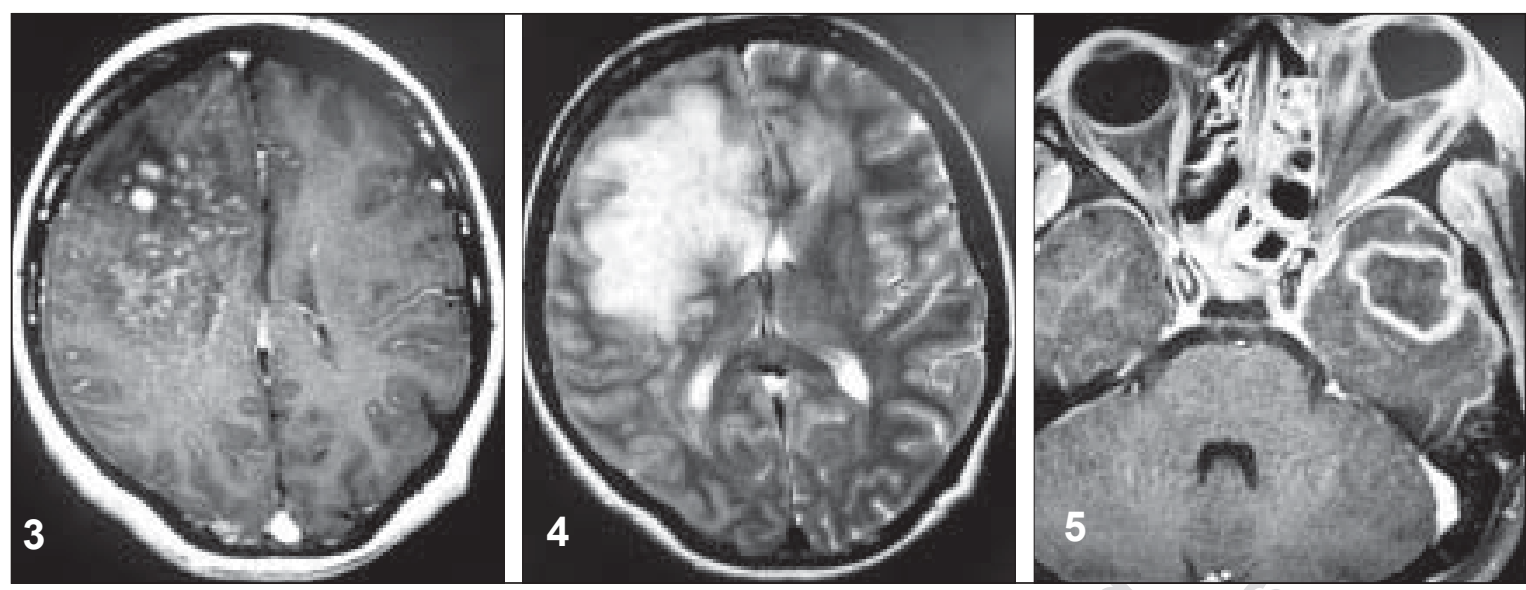

Figures 3, 4, 5: Axial T1 weighted image showing hypo-intense right frontoparietal lesion. T2 weighted image of same showing hyper-intense signals with patchy iso to hypo-intensities. There is significant edema and mass effect. On contrast administration, the lesion showed patchy enhancement, mainly at the center of the lesion. These findings are not typical of aspergillus disease, although the pathology confirmed this to be aspergillosis. Patient was immunocompetent

even no contrast enhancement [Figures 3-5]. Early hemorrhage or infarctions are other features associated with aspergillosis and should raise the suspicion in immunocompromised hosts. Overall, CT scanning not only helps in the diagnosis but is also helpful in providing a convenient way to monitor the patient's response to antimicrobial therapy.

On MR imaging, the classical description is that of an irregular space-occupying mass lesion having hypo-to iso-intense signals on T1-weighted images [Figure 6], extremely low signals on T2-weighted images with either bright homogenous enhancement on postgadolinium $\mathrm{T} 1$ weighted images or ring enhancement pattern. At times a thick irregular wall of mass lesions on CT and MR imaging is apparent, which indicates a competent host defense mechanism that is attempting to isolate or encapsulate the offending organisms. The differential diagnosis of multiple brain lesions in an

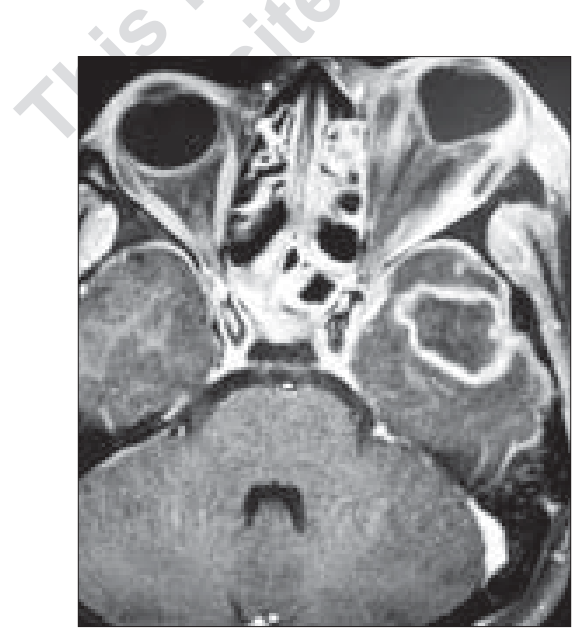

Figure 6: Axial T1 weighted image with gadolinium enhancement showing nasal and left orbital involvement with left proptosis. Patient also has a left temporal ring-enhancing abscess in the absence of bony erosion. The lesions turned out to be Aspergillus in etiology. Patient was immunocompetent immunocompromised patient includes lymphoma, metastatic disease, septic emboli, tuberculomas and multiple infarcts. It may be mentioned here, that the diagnosis of aspergillosis in all cases is supported with the involvement of paranasal sinuses but as for all reported features, it is also not diagnostic on its own.

\section{Surgical Management}

There are no specific treatment guidelines for intracranial aspergillosis, as detailed data on patient survival is only available from case reports and case series. ${ }^{[19,20]}$ Despite newer, safer and more effective antifungal medications, it is generally agreed that the cornerstone of treatment remains surgical excision. This is based largely on reports of personal experience; and more recently on the basis of a multifactorial risk analysis done by Schwartz et al. on the data of a study population comprising mainly immunocompromised patients on variconazole therapy, which suggested improved outcomes in patients who had undergone neurosurgical intervention. ${ }^{[19]}$ Besides, there are very few reports of patients surviving CNS aspergillosis on medical treatment alone. ${ }^{[20,21]}$ It cannot be overemphasized that surgical excision must always be followed by aggressive antifungal chemotherapy to achieve the best response. ${ }^{[19,22-26]}$ It has been observed that radical surgery done early and as a first procedure is better than repeated attempts at sub-radical resection. ${ }^{[27]}$ We have reported our own experience from two large university hospitals of Karachi and in our experience radical excision of fungal mass does not seem to be necessary. ${ }^{[2]}$ Radical surgery involving risks of morbidity seems excessive for an infective process. Sub-radical excision aimed at establishing diagnosis and reducing disease burden, in process, avoiding the risks of permanent iatrogenic neurological deficits, followed by systemic antifungal 
therapy seems to be a better course of action. However, whenever faced with a surgically removable lesion in a noneloquent area, the best plan would be to eradicate the lesion completely rather than sub-radical resection. Sub-radical resection may be reserved for lesions which are only partially resectable. Obviously, in a lesion occupying an eloquent area, where iatrogenic permanent neurological deficits seem inevitable even if sub-radical excision is attempted, a biopsy would suffice.

A similar approach is advisable in case of cerebral aspergillus abscesses. These should be excised completely if there is no risk of further neurological deficits as complete excision has been shown to improve outcome. ${ }^{[28]}$ Even lobectomy, in patients with a single aspergillus abscess is an acceptable surgical option when noneloquent areas of the brain are involved. In the series reported by Nadkarni and Goel, four of seven patients with cerebral aspergillosis who survived, complete surgical resection of brain abscess was accomplished. ${ }^{[1]}$ On the other hand, when eloquent brain areas are found to harbor an aspergillus abscess, aspiration may be the only surgical option which may be done more precisely under neuronavigation or stereotactic guidance, especially if the abscesses are deep-seated. It should be borne in mind that abscess fluid may not be diagnostic in all cases as fungal hyphae are abundant largely in the abscess rim rather than the necrotic center. For best diagnostic yields, multiple specimens from both the rim and center should be sought. This procedure may also be carried out in awake patients under local anesthesia using burr hole or mini craniotomy. Necrotic brain tissue and granulomas are likewise subjected to radical debridement which would theoretically enhance antibiotic penetration and reduce fungal load by removal of debris. Extradural disease is less complicated to deal with and should be treated according to basic neurosurgical principles established for other forms of exradural abscesses, followed of course by antifungal therapy.

Whenever the nasal passages are suspected to be involved, we strongly recommend thorough clearance of nasal passages, thereby depriving the fungus of a potential microaerophilic environment otherwise suitable for its proliferation. For this purpose, we have benefited greatly from endoscopic sinus surgery, which requires the help of ENT surgeons. The procedure may need to be repeated more than once for adequate clearance of nasal passages and sinuses. When the globe is also involved the patient mostly does not have vision in that eye and enucleation should be considered and discussed with the patient. This becomes even more difficult when the globe is clearly affected by the fungus but the vision is intact. In such cases, preservation of the eye with serial monitoring with MRI and objective ophthalmological examinations such as perimetry and visual evoked potentials may be considered. The management therefore requires multidisciplinary team approach involving the neurosurgeon, ENT surgeon, ophthalmologist, maxillofacial surgeon, neuroradiologist, intensivist, infectious disease expert and clinical pharmacist.

Perioperative management of these patients requires special mentioning. It is a critical time period which starts from the time of incision and lasts almost a week or even longer. It is the most difficult part of management and from our experience we have learned that it requires more vigilance and aggression than perhaps the surgery itself. The Aspergillus shows a peculiar response to surgery, not reported for any other disease process. The disease process which may have been present for some time, flares up postoperatively assuming a rapidly fulminant course if not dealt with immediately and aggressively. The hallmark of this unusual response is high-grade fever, tachycardia and rapidly increasing diffuse cerebral edema causing a potentially fatal rise in intracranial pressure refractory to medical management, a series of events that we refer to as the "Forest Fire Phenomenon". The reason for this unique stormy postoperative course is incompletely understood but may be explained on the basis of either the patient becoming immunocompromised secondary to surgery or due to the manipulation of organism catalyzing the stimulation of previously quiescent fungi. This eventually leads to widespread catastrophic angio-invasion causing massive cerebral edema as well as possibly massive release of cytokines. Another possibility is that of acute Aspergillus meningo-encephalitis which may also lead to diffuse cerebral edema. The organism's response to surgery is sometimes apparent even intraoperatively as the patient's body temperature begins to rise, at times beyond 40 degrees and the brain starts to swell up rapidly. We recommend that the surgery for fungal infections be of shortest possible duration, yet as aggressive as possible. At the slightest intraoperative impression of increasing brain swelling, serious thought should be given to keeping the bone flap off and doing duroplasty, thus allowing the brain to further swell up without the risk of herniation. Indeed, we have had to take the patient back to the operating room at times twice within the first $48 \mathrm{~h}$ to deal with the increasing brain swelling which is refractory to medical management. As a result, we now routinely monitor intracranial pressure in these patients postoperatively with an intra-ventricular catheter, keep them in intensive care units and manage them with high doses of Mannitol. The role of steroids is questionable, as hypothetically it would further compromise the patient's immune status but on the other hand, it may help control the edema on virtue of its effect on the vasogenic component of cerebral edema. There is no evidence which supports its use or avoidance and the decision would rest with the neurosurgeon's comfort level. The patient is kept intubated and intermittently sedated using Propofol 
which also helps in decreasing intracranial pressure. Continuous sedation is avoided to allow observation of progressive neurological deficits. Repeat neuro-imaging studies at appropriate intervals may be used to monitor the intracranial complications in the postoperative phase. This helps in comparing the extent of cerebral edema and also in ruling out acute hemorrhage and hydrocephalus, two established postoperative complications as we shall discuss later. At the slightest hint of increasing cerebral edema and impending herniation, a decompressive craniotomy is undertaken. Decompression may just involve removing the bone flap with or without duroplasty, frontal lobectomy, temporal lobectomy with or without uncusectomy or all of the mentioned procedures depending upon the degree of swelling. The craniotomy may need to be extended under such circumstances to increase the size of bone flap which is to be removed. The bone flap may be saved in the patient's anterior abdominal wall which has the advantage of showing early signs of infection and also convenience if the patient needs to be shifted to another hospital. We, however, preserve our bone flaps sterilized in subfreezing temperatures in a dedicated bone bank. We do not see any point in discarding the bone flap and later using acrylic for cranioplasty in such patients, however individual practices may vary. If the bone flap is in close proximity of fungal mass, alternate closure methods may be considered. Autoclaving the bone before putting it back is also an option under such circumstances.

In the postoperative period, special attention should be given to the patient's metabolic profile, as the patient is under the influence of a number of factors which may lead to metabolic derangements and may jeopardize the entire management. These factors include fungal sepsis secondary to manipulation of the fungal mass, high-grade fever and possible risk of acute renal failure due to combined insults from sepsis, mannitol and most importantly the antifungal medications. Electrolyte imbalance may occur acutely and all electrolytes need to be repeated and corrected every two to four hours. Patient is kept on regular high-dose analgesics and anti-pyretic to avoid any increase in intra-cranial pressure (ICP) secondary to pain or fever. We tend to avoid non-steroidal anti-inflammatory drugs for the possible nephrotoxicity and platelet dysfunction despite their superiority in controlling both fever and pain. Continuous invasive blood pressure monitoring using an arterial line is mandatory. The mean arterial pressure (MAP) is kept at around 100-110 as too low may induce infarctions in the swollen brain and too high may further worsen the ICP. Any rapid rise in MAP and ICP should suggest ongoing complications. Fluid balance is also critical as too much fluid may increase cerebral edema and too little may worsen renal failure. A central venous line is therefore essential and a central venous pressure (CVP) of 8-10 is desirable, keeping the patient euvolemic. Persistently low CVP refractory to fluid challenges suggests systemic vasodilatation secondary to fungal sepsis and should be dealt with systemic vasoconstrictors. We generally prefer nor-epinephrine for this which is started at the lowest possible dose and adjusted according to the patient's need. Our choice of fluid was isotonic saline $(0.9 \%)$ as theoretically it has the least effect on ICP. In case of worsening ICP, hypertonic saline may also be considered. The urine output must be maintained at above $1-2 \mathrm{ml} / \mathrm{kg}$ body weight and in case of fall in urine output, rise in blood urea, nitrogen and creatinine levels, hemodyalisis may need to be considered as an alternate to discontinuing nephrotoxic antifungal medications. The multidisciplinary team approach is most important in this timeframe. Cardiac arrhythmias may be precipitated due to both sepsis and electrolyte imbalance and in a patient with a cardiac history or showing abnormal rhythm, a SwanGanz catheter may be required depending upon the internist's decision. The neurosurgery team must always be involved in all decisions, for at times a central complication may manifest as a systemic one and may be missed by those not trained in neuro-intensive care. We routinely keep our patients on anti-epileptic medications starting preoperatively, although there is no evidence to suggest any benefit of prophylactic antiepileptic medications. Our argument for anti-epileptic drugs is based on the extremely critical condition of these patients postoperatively and any factor which may increase the intracranial pressure during this time such as a seizure may prove detrimental. Interestingly, such maneuvers are not required in patients having extradural disease alone. It is only when the fungus involves brain parenchyma and during surgery the dura is opened that we see the "Forest Fire Phenomenon".

\section{Adjunct Medical Management}

The medical management of intracranial fungal infections should not be considered separately from the surgical management. It is complementary to surgery and should be considered an essential component of the overall management. For a long time, the standard therapeutic regimen for cerebral aspergillosis following surgery remained intravenously administered amphotericin B combined with or followed by flucytosine or itraconazole. ${ }^{[2,23-32]}$ Since the introduction of itraconazole, the first azole with significant activity against Aspergillus, many multicenter clinical trials have proven its efficacy, in combination or in sequential regimen. ${ }^{[33-38]}$ Two newer parenteral antifungal medications (echinocandins) caspofungin and micafungin, which were initially studied for their effectiveness against Candidiasis are also under scrutiny. However, all of the aforementioned medications are 
used mainly as adjuncts to surgical management, as despite improving outcomes compared to control groups; these show relatively poor penetration into the CNS. Amphotericin B, itraconazole and caspofungin all show negligible levels in CSF or brain tissue. ${ }^{[39-41]} \mathrm{A}$ newer triazole, voriconazole is becoming increasingly popular and has been shown to have a CSF to plasma ratio of 0.22 to 1 , as well as high brain tissue levels in autopsy studies. ${ }^{[19-42]}$ In several studies and one large randomized trial conducted by Herbrecht et al. recently, voriconazole has proven to be more effective and less toxic than amphotericin $\mathrm{B}$ and has since become the first line of treatment of aspergillosis. ${ }^{[12,43-47]}$ Recently, Clemons et al. supported the potential of combination therapy as a mechanism to improve the outcome in CNS aspergillosis, showing that a combination of suboptimal doses of liposomal amphotericin B and voriconazole given concurrently had a significantly better efficacy in comparison with either monotherapy. ${ }^{[48]}$ In addition, liposomal amphotericin B in combination with caspofungin and micafungin also showed strong trends towards improved efficacy.

As a protocol, we now administer preoperative oral itraconazole empirically in all our patients in whom a diagnosis of cerebral aspergillosis is suspected on the basis of clinical suspicion or neuroradiological features. This prophylactic regimen in our published experience leads to improved outcomes and prolonged survival, even though a direct causal relationship has not been effectively established. ${ }^{[2]}$ Some authors have similarly proposed preoperative amphotericin $\mathrm{B}$ as a potential factor for improving prognosis. ${ }^{[49,50]}$ As for all rare diseases, it is not possible to scientifically comment upon the comparative efficacy of these prophylactic medications based entirely on personal experiences. A systemic review of the literature with the sole purpose of establishing the role of one or the other prophylactic antifungal medication is needed. It may be argued here that although the effectiveness of any of these medications preoperatively is not proven scientifically, they have certainly not been proven to be harmful. Preoperative oral itraconazole as part of the protocol for the management of all such patients should be considered seriously. A few anecdotal reports have been published that describe treatment with intracavitary amphotericin B. ${ }^{[20,21,51,52]}$ In our opinion, this should only be considered as a last resort as there is no evidence of the safety and efficacy of the drug when directly instilled into the ventricles or the abscess cavity.

\section{Complications and Prognosis}

The unpredictable nature of the disease is apparent not only in its clinical presentation, radiological appearance, clinical course and response to treatment, but also when dealing with the postoperative patient.
Besides the general complications anticipated after any major cranial procedure and the adverse effects described for the antifungal medications, some specific complications reported for these patients include massive anaphylaxis, meningitis, mycotic aneurysm formation, intracerebral hemorrhage, subarachnoid hemorrhage and hydrocephalus. Hydrocephalus is usually of the communicating type, although noncommunicating type may also occur. Patients need to be serially monitored with CT scans for this complication which can easily be dealt with ventriculoperitoneal shunt. Perhaps the two most catastrophic complications, both unusual in etiology are; widespread multifocal dissemination in the brain parenchyma/ ventricles and vascular infarcts involving major cerebral vessels, which may be remote from the site of surgery. ${ }^{[2,53-59]}$ Such patients show a stormy postoperative course and may require major debulking or even decompressive craniotomy to prevent herniation due to rapidly increasing intracranial pressures. Despite these efforts, patients may still succumb to the disease and those who survive, will have crippling neurological deficits. Both of these complications are attributable to subclinical or occult angioinvasion, which is a hallmark of the disease as discussed in previous sections.

Outcome of craniocerebral aspergillosis is different in immunosuppressed and apparently immunocompetent hosts. ${ }^{[23,29]}$ In immunocompromised hosts mortality is reported to reach more than 95\% and even $100 \%$ in patients with underlying malignancies. ${ }^{[60]}$ In apparently immunocompetent hosts reported mortality varies from center to center, but ranges from $40-80 \% .^{[1,2]}$ Patients harboring mainly sinonasal disease with only orbital and/or cranial base bony invasion/destruction, carry a very good prognosis (mortality $<20 \%$ ). ${ }^{[2]}$ Outcome in extradural aspergillosis is much better than in patients with intracerebral involvement. ${ }^{[2]}$ The dismal prognosis should nevertheless, not discourage the care providers from aggressive management of these patients as success stories are also frequently encountered.

\section{Conclusion}

Aspergillusinfection of the CNS is an uncommon clinical entity, affecting mainly the immunocompromised. The disease presents in a number of unusual ways and although a multitude of clinical, biochemical and radiological features have been described, the diagnosis remains elusive. The problem is further compounded when the host is apparently immunocompetent, which lowers the clinical suspicion. Surgery followed by systemic antifungal medications offers the best hope for survival. Despite better understanding of the offending organism, pathophysiology, clinical course and development of more effective antifungal medications, the prognosis remains poor. High index of suspicion, 
early diagnosis and aggressive surgical management, followed by medical therapy, by an interdisciplinary team, may help to improve outcomes.

\section{References}

1. Nadkarni T, Goel A. Aspergilloma of the brain: An overview. J Postgrad Med 2005;51:37-41.

2. Siddiqui AA, Shah AA, Bashir SH. Craniocerebral asergillosis of sino-nasal origin in immunocompetent patients: Clinical spectrum and outcome in 25 cases. Neurosurgery 2004;55:602-13.

3. Walsh T.J, Hier DB, Caplan LR. Aspergillosis of the central nervous system: Clinicopathological analysis of 17 patients. Ann Neurol 1985; 18:574-82.

4. Artico M, Pastore FS, Polosa M, Sherkat S, Neroni M. Intracerebral Aspergillus abscess: Case report and review of the literature. Neurosurg Rev 1997;20:135-8.

5. Jamjoom AB, al-Hedaithy SA, Jamjoom ZA, al-Hedaithy M, el-Watidy SF, Rahman N, et al. Intracranial mycotic infections in neurosurgical practice. Acta Neurochir (Wien) 1995;137:78-84.

6. Das SK, Bhattacharya S, Mitra K, Roy T, Bhattacharya M, Ghorai SP, et al. Central nervous system fungal infection: A report of three cases. J Indian Med Assoc 2004;102:634-6.

7. Walsh TJ, Hier DB, Caplan LR. Fungal infections of the central nervous system: Comparative analysis of risk factors and clinical signs in 57 patients. Neurolgy 1985;35:1654-7.

8. Levy RM, Bredsen DE, Rosenblum ML. Neurological manifestations of the acquired immuno deficiency syndrome (AIDS) experience at UCSF and review of literature. J Neurosurg 1985;62:475-95.

9. Alapatt JP, Kutty RK, Gopi PP, Chalissery J. Middle and posterior fossa aspergilloma. Surg Neurol 2006;66:75-9.

10. Speth C, Rambach G, Lass-Flörl C, Würzner R, Gasque P, Mohsenipour I, et al. Culture supernatants of patient-derived Aspergillus isolates have toxic and lytic activity towards neurons and glial cells. FEMS Immun Med Microbiol 2000;29:303-13.

11. Sharma RR, Lad SD, Desai AP, Lynch PG. Surgical management of fungal infections of the nervous system. In: Schmidek HH, editor, Schmidek and Sweet Operative Neurosurgical Techniques: Indications, Methods and Results, $4^{\text {th }}$ ed. WB Saunders Company: Philadelphia; 2000,.p.1726-55.

12. Stevens DA, Kan VL, Judson MA, Morrison VA, Dummer S, Denning DW, et al. Practice guidelines for diseases caused by Aspergillus. Infectious Diseases Society of America. Clin Infect Dis 2000;30:6967 .

13. Beal MF, O'Carroll P, Kleinman GM, Grossman RI. Aspergillosis of the nervous system. Neurology 1982;32:473-9.

14. Jinkins JR, Siqueira E, Al-Kawi MZ. Cranial manifestations of aspergillosis. Neuroradiology 1987;29:181-5.

15. Hall WA. Neurosurgical infections in the compromised host. In: Haines SJ, Hall WA, editors, Neurosurgery Clinies of North America, Infections in Neurologic Surgery, Vol 3, WB Saunders Co: Philadelphia; 1992. p. 435-42.

16. MacCormick WF, Schochet SS Jr, Weaver PR, MacCrary JA $3^{\text {rd }}$. Disseminated aspergillosis. Aspergillus endophthalmitis, optic nerve infarction, and carotid artery thrombosis. Arch Pathol 1975;99:3539.

17. Sorensen AG, Buonanno FS, Gonzalez RG, Schwamm LH, Lev MH, Huang-Hellinger FR, et al. Hyperacute stroke: Evaluation with combined multi section diffusion-weighted and hemodynamically weighted echo-planar MR imaging. Radiology 1996;199:391-401.

18. Boes B, Bashir R, Boes C, Hahn F, McConnell JR, McComb R. Central nervous system aspergillosis analysis of 26 patients. J Neuroimaging 1994;4:123-9.

19. Schwartz S, Ruhnke M, Ribaud P, Corey L, Driscoll T, Cornely OA, et al. Improved outcome in central nervous system aspergillosis, using voriconazole treatment. Blood 2005;06:2641-5.

20. Coleman JM, Hogg GG, Rosenfeld JV, Waters KD. Invasive central nervous system aspergillosis: Cure with liposomal amphotericin B, itraconazole and radical surgery: A case report and review of the literature. Neurosurgery 1995;36:858-63.

21. Ellis M, Watson R, MeNabb A, Lukic ML, Nork M. Massive intracerebral aspergillosis responding to combination high dose liposomal amphotericin B and cytokine therapy without surgery. J Med Microbiol 2002;51:705.

22. Alrajhi AA, Enani M, Mahasin Z, Al-Omran K. Chronic invasive aspergillosis of the paranasal sinuses in immunocompetent hosts from Saudi Arabia. Am J Trop Med Hyg 2001;65:83-6.

23. Denning DW. Therapeutic outcome in invasive aspergillosis. Clin Infect Dis 1996;23:608-15.

24. Kontoyiannis DP, Bodey GP. Invasive aspergillosis in 2002: An update. Eur J Clin Microbiol Infect Dis 2002;21:161-72.

25. Steinbach WJ, Stevens DA, Denning DW. Combination and sequential antifungal therapy for invasive aspergillosis: Review of published in vitro and in vivo interactions and 6281 clinical cases from 1966 to 2001. Clin Infect Dis 2003;37:S188-224.

26. Young RF, Gade G, Grinnel V. Surgical treatment for fungal infections in the central nervous system. J Neurosurg 1985;63:371-81.

27. Naim-Ur-Rahman, Jamjoom A, al-Hedaithy SS, Jamjoom ZA, alSohaibani MO, Aziz SA. Cranial and intracranial aspergillosis of sinonasal origin: Report of nine cases. Acta Neurochir (Wien) 1996;138:94450 .

28. Murthy JM, Sundaram C, Prasad VS, Purohit AK, Rammurti S, Laxmi V. Aspergillosis of central nervous system: A study of 21 patients seen in a university hospital in south India. J Assoc Physicians India 2000;48:677-81

29. Denning DW, Stevens DA. Antifungal and surgical treatment of invasive aspergillosis: Review of 2121 published cases. Rev Infect Dis $1990 ; 12: 1147-201$

30. Kim DG, Hong SC, Kim HJ, Chi JG, Han MH, Choi KS, et al. Cerebral aspergillosis in immunologically competent patients. Surg Neurol 1993;40:326-31.

31. Kurita H, Shiokawa Y, Furuya K, Segawa H, Sano K. Parasellar Aspergillus granuloma extending from the sphenoid sinus: Report of two cases. Surg Neurol 1995;44:489-94.

32. Sobel JD. Practice guideline for the treatment of fungal infections. For the Mycoses Study Group. Infectious Diseases Society of America. Clin Infect Dis 2000;30:652-6.

33. De Beule K, Gestel JV. Pharmacology of itraconazole. Drugs 2001;61:2737.

34. Denning DW, Lee JY, Hostetler JS, Pappas P, Kauffman CA, Dewsnup DH, et al. NIAID mycoses study group multicenter trail of oral itraconazole therapy for invasive aspergillosis. Am J Med 1994;97:13544.

35. Mikolich DJ, Kinsella LJ, Skowron G, Friedman J, Sugar AM. Aspergillus meningitis in immunocompetent adults successfully treated with itraconazole. Clin Infect Dis 1996;23:1318-9.

36. Harousseau JL, Dekker AW, Stamatoullas-Bastard A, Fassas A, Linkesch W, Gouveia J, et al. Itraconazole oral solution for primary prophylaxis of fungal infections in patients with hematological malignancy and profound neutropenia: A randomized, doubleblind, double-placebo, multicenter trial comparing itraconazole and amphotericin B. Antimicrob Agents Chemother 2000;44:1887-93.

37. Rowe-Jones JM, Freedman AR. Adjuvant itraconazole in the treatment of destructive sphenoid aspergillosis. Rhinology 1994;32:203-7.

38. Sanchez C, Mauri E, Dalmau D, Quintana S, Apatricio A, Garau J. Treatment of cerebral aspergillosis with itraconazole: Do high doses improve the prognosis? Clin Infect Dis 1995;21:1485-7.

39. Golin V, Sprovieri SR, Cançado JE, Daniel JW, Mimica LM. Aspergillosis of the central nervous system. Sao Paulo J Med 1996;114:1274-7.

40. Satoh H, Uozumi T, Kiya K, Ikawa F, Kurisu K, Sumida M, et al. Invasive aspergilloma of the frontal base causing internal carotid artery occlusion. Surg Neurol 1995;44:483-8.

41. Heykants J, Michiels M, Meuldermans W, et al. The pharmacokinetics of itraconazole in animals and man: An overview. In: Fromtling RA, editor. Recent trends in the discovery, development and evaluation of antifungal agents. JR Prous Science Publishers: Barcelona, Spain; 1987. p. $223-49$

42. Collette N, van der Auwera P, Lopez AP, Heymans C, Meunier F. 
Tissue concentrations and bioactivity of amphotericin B in cancer patients treated with amphotericin B deoxycholate. Antimicrob Agents Chemother 1989;33:362-8.

43. Collette N, van der Auwera P, Meunier F, Lambert C, Sculier JP, Coune A. Tissue distribution and bioactivity of amphotericin $\mathrm{B}$ administered in liposomes to cancer patients. J Antimicrob Chemother 1991;27:53548.

44. Lutsar I, Roffey S, Troke P. Voriconazole concentrations in the cerebrospinal fluid and brain tissue of guinea pigs and immunocompromized patients. Clin Infect Dis 2003;37:728-32.

45. Herbrecht R, Denning DW, Patterson TF, Bennett JE, Greene RE, Oestmann JW, et al. Voriconazole versus amphotericin B for primary therapy of invasive aspergillosis. N Engl J Med 2002;347:408-15.

46. Chandrasekar PH, Cutright JL, Manavathu EK. Efficacy of voriconazole plus amphotericin B or micafungin in a guinea-pig model of invasive pulmonary aspergillosis. Clin Microbiol Infect 2004;10:925-8.

47. Kirkpatrick WR, McAtee RK, Fothergill AW, Rinaldi MG, Patterson TF. Efficacy of voriconazole in a guinea pig model of disseminated invasive aspergillosis. Antimicrob Agents Chemother 2000;44:2865-8.

48. Clemons KV, Espiritu M, Parmar R, Stevens DA. Comparative efficacies of conventional amphotericin B, liposomal amphotericin B (ambisome), caspofungin, micafungin and voriconazole alone and in combination against experimental murine central nervous system aspergillosis. Antimicrob Agents Chemother 2005;49:4867-75.

49. Chiller TM, Sobel RA, Luque JC, Clemons KV, Stevens DA. Efficacy of amphotericin B or itraconazole in a murine model of central nervous system Aspergillus infection. Antimicrob Agents Chemother $2003 ; 47: 813-5$.

50. Chiller TM, Stevens DA. Treatment strategies for Aspergillus infections. Drug Resist Updat 2000;3:89-97.

51. Camarata PJ, Dunn DL, Farney AC, Parker RG, Seljeskog EL. Continual intracavitary administration of amphotericin $B$ as an adjunct in the treatment of Aspergillus brain abscess: Case report and review of the literature. Neurosurgery 1992;31:575-9.
52. Elgamal EA, Murshid WR. Intracavitary administration of amphotericin $\mathrm{B}$ in the treatment of cerebral aspergillosis in a non immunocompromized patient: Case report and review of the literature. Br J Neurosurg $2000 ; 14: 137-41$

53. Haran RP, Chandy MJ. Intracranial Aspergillus granuloma. Br J Neurosurg 1993;7:383-8.

54. Goel A, Nadkarni T, Desai AP. Aspergilloma in the paracavernous region. Two case reports. Neurol Med Chir (Tokyo) 1996;36:733-6.

55. Nadkarni TD, Desai KI, Muzumdar D, Goel A, Shenoy A. Ischaemic complications after surgical resection of intracranial aspergilloma. J Clin Neurosci 2003;10:500-2.

56. Endo T, Tominaga T, Konno H, Yoshimoto T. Fatal subarachnoid hemorrhage, with brainstem and cerebellar infarction, cause by Aspergillus infection after cerebral aneurysm surgery: Case report. Neurosurgery 2002;50:1147-51.

57. Takeshita M, Izawa M, Kubo O, Tanikawa T, Onda H, Wanifuchi H, et al. Aspergillotic aneurysm formation of cerebral artery following neurosurgical operation. Surg Neurol 1992;38:146-51.

58. Viriyavejakul P, Phudhichareonrat S, Boonpasat Y, Viriyavejakul A, Rojanasunan P, Phophak N, et al. Intracerebral hemorrhage due to nosocomial aspergillosis following neurosurgery. Southeast Asian J Trop Med Public Health 1999;30:154-6.

59. Aslam E, Imran M, Muhammad T, Faridi N. Rhinocerebral aspergillosis cripple: High oral doses of Itraconazole was the solution. J Pak Med Assoe 2006;56:415-7.

60. Chernik NL, Armstrong D, Posner JB. Central nervous system infections in patients with cancer. Medicine (Baltimore) 1973;52:56381.

Accepted on 29-06-2007

Source of Support: Queensland Health Department 